\title{
COVID-19 and liver injury: where do we stand?
}

\author{
Nikolaos Papadopoulosa, ${ }^{a, b}$ Sofia Vasileiadi' ${ }^{\text {, Melanie Deutsch }}{ }^{\mathrm{b}}$
}

417 Army Share Fund Hospital of Athens; Hippokration General Hospital of Athens, Medical School of National and Kapodistrian University of Athens, Greece

Abstract

The coronavirus SARS-CoV-2 was identified as the cause of COVID-19, a severe acute respiratory syndrome. Several clinical studies refer to liver injury as the most frequent clinical extrapulmonary manifestation. In this review, we summarize the available clinical data concerning liver injury during COVID-19. Although the underlying mechanism of liver impairment is somewhat unclear, transaminases and bilirubin levels are elevated in a substantial proportion of patients. Moreover, more severe alterations in liver enzymes may correlate with a worse clinical course of COVID-19. However, several other cofactors, such as drug-induced liver injury, hyper-inflammatory response to infection, hypoxic hepatitis or preexisting underlying liver disease, cannot be excluded.

Keywords Coronavirus, SARS-CoV-2, COVID-19, liver injury

Ann Gastroenterol 2020; 33 (4): 1-6

\section{Introduction}

At the end of 2019, a novel coronavirus (SARS-CoV-2) was identified as the cause of a severe acute respiratory syndrome (coronavirus disease 2019, COVID-19) in Wuhan, a city in the Hubei Province of China. It rapidly spread as an epidemic throughout China, finally resulting in a worldwide pandemic [1]. According to the European Centre for Disease Prevention and Control, up to 02 June 2020 a total of 6,245,352 cases of COVID-19 have been reported, including 376,427 deaths [2].

Pneumonia and respiratory failure appear to be the most frequent serious manifestations of COVID-19, while the spectrum of illness severity ranges from mild (most cases) to critical disease, with an overall case fatality rate of $2.3 \%[3,4]$. However, despite its "respiratory preference", COVID-19 can be recognized as a systemic disease with multiple organ involvement. In this context, recently published data have described abnormal liver function tests, mainly elevated alanine aminotransferase (ALT) and aspartate aminotransferase (AST),

${ }^{\mathrm{a}} 1$ st Department of Internal Medicine, 417 Army Share Fund Hospital of Athens (Nikolaos Papadopoulos); b2nd Department of Internal Medicine, Hippokration General Hospital of Athens, Medical School of National \& Kapodistrian University of Athens (Sofia Vasileiadi, Melanie Deutsch), Greece

Conflict of interest: None

Correspondence to: Nikolaos Papadopoulos, Monis Petraki 10-12, 11521 Athens, Greece, e-mail: nipapmed@gmail.com

Received 19 April 2020; accepted 19 May 2020;

published online 22 June 2020

DOI: https://doi.org/10.20524/aog.2020.0522 as the most common extrapulmonary finding in COVID-19 cases $[5,6]$.

The exact underlying pathophysiology and the clinical implication of COVID-19-associated liver injury have not yet been fully determined, while much research regarding this issue is ongoing. Based on previous experience, the role of coronaviruses in generating liver injury has been documented in patients infected by the SARS-CoV coronavirus, described as an acute respiratory syndrome back in 2003 [7]. While the presence of SARS-CoV-2 has been confirmed in liver tissue, it is unclear whether the liver injury can be caused directly by the coronavirus itself [8-10]. Interestingly, both SARS-CoV-2 and SARS-CoV bind to the angiotensin-converting enzyme 2 (ACE2) receptor to enter the target cell [8]. However, while it was demonstrated that the ACE2 receptor is expressed in the biliary epithelial liver cells, liver injury in COVID-19 patients is mainly revealed as hepatocyte injury documented by ALT and AST elevation [11]. Thus, an alternative pathophysiology of liver injury may be implicated in these patients $[5,12]$. One hypothesis is that it may be the result of the characteristic immune-mediated damage and systemic inflammation caused by a hyper-activated response to COVID-19, described in patients with severe clinical manifestations [12,13]. Other possible explanations and alternative mechanisms, such as drug-induced liver damage (DILI) associated with paracetamol, antivirals (remdesivir, lopinavir/ritonavir), antimicrobials (macrolides, quinolones, beta-lactams, chloroquine), or nonsteroidal anti-inflammatory drugs (NSAIDs), and hypoxic hepatitis due to respiratory or cardiac failure in severe cases, cannot be ruled out $[10,12]$.

This review aims to summarize the available data concerning the extent and the role of liver injury as an extrapulmonary manifestation of COVID-19. A literature search in the PubMed database was conducted until 16 April 2020 for studies of 
COVID-19. A keyword-base search included the entries "COVID-19" OR "nCOV" OR "novel-coronavirus OR "SARSCoV-2 AND "liver" OR "transaminases" OR "gastrointestinal". We excluded 2 studies published in Chinese. Finally, we included 22 studies with characteristics of liver injury (liver test abnormalities) in COVID-19 patients.

\section{Clinical studies of liver injury in COVID-19 patients}

The first report of liver injury due to COVID-19 was by Chen et al [3]. In a total of 99 Chinese patients who had COVID-19, with a mean age of $55.5 \pm 13.1$ years, the majority of them being male, mild elevations of AST and ALT were recorded in $35 \%$ and $28 \%$, respectively. Only one patient experienced a marked elevation of transaminase levels (ALT $7590 \mathrm{U} / \mathrm{L}$ and AST 1445 U/L). Moreover, albumin levels were decreased in $98 \%$ of the patients, while total bilirubin was increased in $18 \%$. However, $50 \%$ of the patients had chronic diseases, with $11 \%$ reported as having chronic digestive diseases; it was not clear whether these patients had underlying liver disease. The significance of the aminotransferase elevation was not analyzed further and remained unclear, while there was no comment about the clinical course and the outcome of the patient who presented with acute hepatitis.

Another study from China described retrospectively the clinical course of COVID-19 in 62 patients. In this cohort, $16.1 \%$ of the patients experienced AST elevation $\geq 40$ U/L [14]. However, the authors mentioned that $11 \%$ of the patients had preexisting liver disease. A further study of radiological findings from 81 patients with COVID-19 revealed that more than half of them (53\%) also had mildly elevated AST levels [15].

In the first large multicenter study, which included 1099 patients from 522 hospitals in China with laboratory-confirmed COVID-19 (median age 47 years, $42 \%$ female, $23.7 \%$ having at least one coexisting illness), abnormal liver function tests (AST, ALT and total bilirubin) were recorded in $22.2 \%, 21.3 \%$ and $10.5 \%$ of the cases, respectively [16]. No further data were available in this report regarding the impact of the abnormal liver function tests on the patients' general clinical course.

In general, most of the studies from China describe a prevalence of abnormal liver function tests of $20-50 \%$. However, recently published data among 80 imported cases (not Wuhan) of COVID-19 revealed that, compared to cases in Wuhan, liver dysfunction is uncommon (3.75\%) [17].

\section{Clinical studies of liver injury in critically ill COVID-19 patients}

In a retrospective single-center study of 138 confirmed patients with pneumonia due to COVID-19, the median AST and ALT levels were reported to be significantly higher in patients who had been treated in the intensive care unit (ICU), rather than in non-ICU beds: 52 (range: 30-70) U/L vs. 29 (21-38) U/L, $\mathrm{P}<0.001$; and 35 (19-57) U/L vs. 23 (15-36) U/L,
$\mathrm{P}=0.007$, respectively. However, in this cohort, the percentage of patients with AST/ALT elevation was not provided [8].

Similarly, in a recently published cohort of 41 patients with laboratory-confirmed COVID-19, 37\% of the patients presented AST elevation, while the median levels of ALT and total bilirubin were 32 (21-50) U/L and $0.68(0.56-0.81) \mathrm{mg} / \mathrm{dL}$, respectively [18]. In accordance with previous reports, the authors also revealed that ICU patients experienced slightly higher levels of AST, ALT and total bilirubin than non-ICU patients.

Cai et al reported that $8.7 \%$ of 298 patients infected by COVID-19 presented abnormal AST and ALT levels [19]. Cholestatic enzymes, such as $\gamma$-glutamyl transferase/alkaline phosphatase (GGT/ALP), were also slightly elevated in a few patients $(3.1 \%$ and $3.0 \%)$. The incidence of liver injury in severe patients $(36.2 \%)$ was markedly higher than that in mild patients (9.6\%).

A retrospective study of the clinical characteristics of 128 laboratory-confirmed COVID-19 cases did not harmonize with previous findings, since AST/ALT levels did not differ significantly between severe and non-severe cases: (28.89 $\pm 31.83 \mathrm{U} / \mathrm{L}$ vs. $43.87 \pm 47.8 \mathrm{U} / \mathrm{L}, \mathrm{P}>0.05$; and $27.98 \pm 25.8$ $\mathrm{U} / \mathrm{L}$ vs. $44.13 \pm 36.26 \mathrm{U} / \mathrm{L}, \mathrm{P}>0.05$, respectively [20].

In a single-center study that included 52 critically ill adult patients with COVID-19 pneumonia admitted to the ICU, liver dysfunction was recorded in 15 (29\%) of them [21]. Finally, 32 $(61.5 \%)$ patients died, but no difference was recorded in the incidence of liver dysfunction between survivors (30\%) and non-survivors $(28 \%)$. However, in this paper, the term "liver dysfunction" is not clearly defined by the authors. Thus, the proportion of patients with abnormal AST, ALT, total bilirubin and prothrombin time (PT) status is not mentioned.

The evaluation of possible prognostic factors for the presence of refractory COVID-19 was assessed in a very recent retrospective study that included a total of 155 patients. Eighty-five patients were classified as "refractory", defined as not reaching obvious clinical and radiological remission within 10 days after hospitalization [22]. Only AST levels were significantly higher in refractory cases: 37 (25-65) vs. 32 (2338), $\mathrm{P}=0.004$. However, multivariate analysis revealed that only male sex $(\mathrm{P}=0.047$; odds ratio $[\mathrm{OR}] 2.206,95 \%$ confidence interval [CI] 1.012-4.809) and anorexia on admission $(\mathrm{P}=0.030$; OR 3.921, 95\%CI 1.144-13.443) were risk factors for disease refractoriness.

To identify risk factors for death in patients with COVID-19, a retrospective cohort study included 191 patients (54 nonsurvivors and 137 survivors) [23]. ALT levels were statistically significantly different between non-survivors and survivors: 40 (24-51) vs. 27 (15-40), $\mathrm{P}=0.005$. However, multivariate analysis revealed that in-hospital mortality was associated only with older age (OR 1.1, 95\%CI 1.03-1.17, per year increase; $\mathrm{P}=0.0043)$, with a higher sequential organ failure assessment (SOFA) score (OR 5.65, 95\%CI 2.61-12.23; $\mathrm{P}<0.0001$ ), and with a d-dimer test above $1 \mu \mathrm{g} / \mathrm{mL}$ (OR 18.42, 95\%CI 2.64128.55; $\mathrm{P}=0.0033$ ) on admission.

Apart from Chinese studies, data from the first patients with COVID-19 admitted to 2 hospitals in New York City were published very recently [24]. This retrospective case 
series included 393 patients, of whom 130 (33\%) developed respiratory failure leading to invasive mechanical ventilation. Liver cirrhosis was recorded in 6/393 (1.5\%) of the patients. Regarding laboratory findings, AST and AST elevation ( $>40 \mathrm{U} / \mathrm{L}$ ) were present in $46.5 \%$ and $32 \%$ of the patients respectively, while total bilirubin elevation $(>1 \mathrm{mg} / \mathrm{dL})$ was documented in $9.1 \%$. The patients who required mechanical ventilation were more likely to have elevated liver function tests than patients with no invasive mechanical ventilation (AST $63 \%$ vs. $38 \%$, ALT $37.5 \%$ vs. $29.1 \%$, total bilirubin $14.1 \%$ vs. $6.5 \%)$. Eventually, 40 (10.2\%) patients died, but no death was due to liver failure (obviously not even cirrhotic patients).

\section{Clinical studies dealing with general gastrointestinal (GI) symptoms and especially with COVID-19-induced liver injury}

Recently, Luo et al evaluated a large cohort of 1141 patients with COVID-19, focusing on the GI symptoms, finally recorded in $183(16 \%)$ patients. As previously described, a mild increase in serum aminotransferases was noted: AST 65.8 \pm 12.7 U/L and ALT 66.4 \pm 13.2 U/L [25].

Very recently, researchers from the Shanghai Public Health Clinical Center included in a retrospective study (which is still in press) 148 confirmed COVID-19 cases from January 20 to January 31,2020 [26]. The aim of this study was to characterize COVID-19-related liver damage. Their analysis revealed that the $50.7 \%$ of patients who presented with abnormal liver functions at admission were more likely to be male $(62.67 \%$ vs. $38.36 \%, \mathrm{P}=0.005)$ and had a higher fever ( $44 \%$ vs. $27.4 \%$, $\mathrm{P}=0.035$ ). Moreover, the utilization rate of lopinavir/ritonavir after admission was significantly higher in patients with emerging liver injury than in patients with normal liver tests. However, the only significant impact of the presence of liver injury was a relatively prolonged length of hospital stay.

In the same context, the first published study is a retrospective study of 79 non-ICU hospitalized patients with COVID-19 at the Jinyintan Hospital from February 2 to February 23, 2020, comparing their clinical characteristics in relation to liver injury [6]. In order to evaluate the role of SARSCoV-2 in liver injury, the authors did not include patients with viral hepatitis, alcoholic liver disease, liver malignancy or other known chronic liver disease. Although ALT and AST levels were elevated in $31.6 \%$ and $35.4 \%$ of the cases, respectively, their median values were $<3 \times$ the upper limit of normal (ULN) at baseline. Moreover, the median value of bilirubin was 0.74 (0.47-0.9) $\mathrm{mg} / \mathrm{dL}$, with normal ALP and GGT. Trying to assess some correlations, logistic regression analyses in this study revealed that the extent of pulmonary lesions on computed tomography was a predictor of liver function damage (OR 5.265, 95\%CI 1.025-1.371, $\mathrm{P}=0.022$ ).

Another retrospective study enrolled a large number of patients $(\mathrm{N}=651)$, of whom $74(11.4 \%)$ presented with GI symptoms [27]. Liver dysfunction was more common in the group of GI patients than in the non-GI patients: 13/74 (17.57\%) vs. $51 / 577$ ( $8.84 \%)$, respectively, $\mathrm{P}=0.035$. Interestingly, only AST levels were statistically significantly higher in GI patients than in non-GI patients: $29.35(20.87-38.62)$ vs. 24.4 (19.032.0 ), respectively, $\mathrm{P}=0.02$. However, multivariate analysis of risk factors for the severe/critical patients with COVID-19 with GI symptoms did not indicate AST/ALT as a significant variable.

In accordance with these results, Pan et al recently evaluated the clinical characteristics of COVID-19 patients with GI symptoms [28]. Among 204 patients (age 52.9 \pm 16 years, 107 men), about half of them (103 patients; 50.5\%) reported a GI symptom, while AST/ALT elevations were recorded in less than $15 \%$ of the patients. Some indexes of liver function, namely AST, ALT and PT, were significantly higher in GI patients than in non-GI patients $(35.12 \pm 26.58$ vs. $27.48 \pm 23.98, \mathrm{P}=0.032 ; 42.24 \pm 43.83$ vs. $29.53 \pm 23.58, \mathrm{P}=0.011$; $13.13 \pm 1.88$ vs. $12.53 \pm 1.89, \mathrm{P}=0.024$, respectively). Although digestive symptoms became more pronounced in accordance with COVID-19 severity, there was no significant difference in discharge time, days of intensive care or mortality between GI and non-GI patients.

Cai et al recently evaluated the clinical characteristics of 417 laboratory-confirmed COVID-19 patients with abnormal liver tests [29]. Liver test abnormality was defined by the elevation of ALT $>40 \mathrm{U} / \mathrm{L}$, AST $>40 \mathrm{U} / \mathrm{L}$, ALT and total bilirubin $>1 \mathrm{mg} / \mathrm{dL}$, while patients with ALT and/or AST $>3 x$ ULN were classified as having hepatocyte-type liver injury. The authors compared liver function tests on admission and during hospitalization concerning COVID-19 severity. On admission, 170 (41\%) patients had abnormal liver test results, while $33(5 \%)$ had signs of hepatocyte-type liver injury. The presence of abnormal liver tests became more pronounced during hospitalization within 2 weeks, while $26.7 \%$ of the patients progressed to severe pneumonia. Moreover, patients with hepatocyte-type injury or mixed-type injury (the combination of both ALT/AST $>3 \times$ ULN and GGT/ALP $>2 \times$ ULN) at admission had higher odds of progressing to severe pneumonia (OR 2.73, 95\%CI 1.19-6.3, and OR 4.44, 95\%CI 1.93-10.23, respectively). However, during hospitalization, the use of lopinavir/ritonavir was correlated with liver injury (OR 4.44 to 5.03 , both $\mathrm{P}<0.01$ ), indicating a possible DILI as cofactor. Moreover, a liver biopsy from one patient who died showed that liver function impairment during hospitalization could be partly due to the drugs used for treatment, or due to sepsis and shock. The authors concluded that patients with abnormal liver tests had higher risks of progressing to severe disease, but the detrimental effects on the liver were mainly related to certain medications used during hospitalization.

Preliminary results from a multicenter cohort with 70 patients (67 non-severe disease) with COVID-19 indicated liver injury (ALT, AST or bilirubin above normal value) on admission in $45.71 \%$ of them [30]. The hospitalization days were not statistically significantly different between patients with or without liver injury: 16 (12-20) vs. 15 (10-22.5), $\mathrm{P}=0.81$. Since the study population did not receive any medication before admission, the liver injury in these patients was probably caused by a coronavirus-mediated mechanism. 


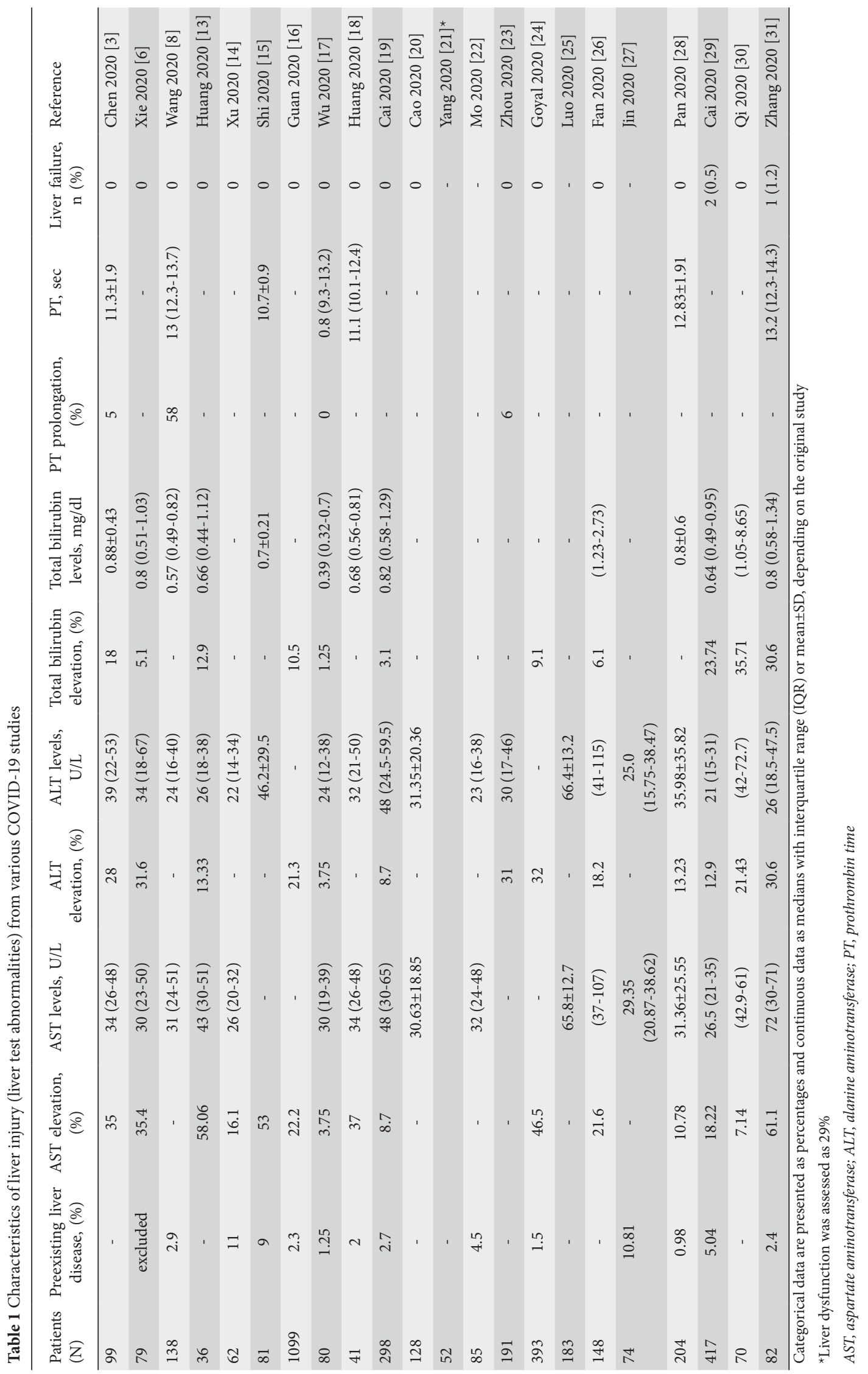




\section{Data concerning liver injury in deceased cases of COVID-19}

These data are supported by 2 studies so far. The first was performed by Zhang et al, who presented an interesting study that evaluated the clinical characteristics of 82 death cases with COVID-19 [31]. Respiratory failure was the leading cause of death $(69.5 \%)$, followed by sepsis syndrome/multiple organ dysfunction syndrome (28.0\%), while liver injury was recorded in $78 \%$ of the patients. One patient died from liver failure, while the authors observed a significant association between AST, ALT levels and time from initial symptom to death. The other study with 36 non-survivors due to confirmed COVID-19 revealed elevation of ALT and AST in $13.33 \%$ and $58.06 \%$ of the patients, respectively [13].

\section{Concluding remarks}

In this review, we have summarized the recent reports of COVID-19-related liver injury, data that are obviously changing rapidly day by day. Although the pathophysiology behind liver injury in COVID-19 remains unresolved, several theories have been proposed, but a multifactorial mechanism seems most likely. Undoubtedly, the available clinical data so far show that the incidence of liver impairment in COVID-19 ranged from 6-61\%, mainly indicated by abnormal ALT/AST levels (Table 1). However, most of the cases experienced a mild AST/ALT elevation (usually $<3 \times$ ULN), accompanied by slightly elevated bilirubin levels. Since ACE2 receptors are expressed especially in the bile ducts, one may suppose that patients should have some degree of cholestasis or jaundice. However, this seems not to be the case in everyday practice and, remarkably, even ICU patients did not present with elevated ALP/GGT.

Although the rate of developing liver injury was higher in patients with severe clinical manifestations of COVID-19 than in mild patients, these differences were statistically nonsignificant in all studies. Moreover, the role of liver dysfunction as a potent predictor of severe COVID-19 manifestation or death was not documented by all selected studies [23,24]. However, in a recently published study, patients with abnormal liver tests had a higher risk of progressing to severe disease [29]. Moreover, in a death case study, one patient died from liver failure and a significant association was revealed between AST and ALT levels and time from initial symptom to death [31].

The possibility of DILI during hospitalization, and other cofactors, such ashypoxichepatitis, as causes ofliver dysfunction, or rhabdomyolysis as a cause of elevated liver enzymes cannot be easily ruled out in clinical practice $[29,32]$. There are only limited available data linking underlying liver diseases with the course of SARS-CoV-2 infection (pre-existing liver disease ranged from $2-11 \%$ in the available data). The European Association for the Study of the Liver and the European Society of Clinical Microbiology and Infectious Diseases have issued a position paper, providing recommendations for clinicians who care for patients with chronic liver diseases during the current
COVID-19 pandemic [33]. This position paper promotes telemedicine in the outpatient setting and suggests avoiding nosocomial dissemination. Additionally, the paper provides recommendations to prevent acetaminophen overdosing, to prevent administration of any NSAID, to continue treatment for cirrhosis-associated complications, to avoid reducing immunosuppressive therapy in patients with autoimmune liver diseases, to include testing for SARS-CoV-2 in patients with acute decompensation or acute on chronic liver failure, and to consider early admission according to the presence of additional risk factors.

In conclusion, the experience so far of clinicians and researchers worldwide suggests that attention should be paid to liver function in all patients infected by COVID-19, but slight/ moderate abnormalities are likely to be of small importance. However, during the course of the disease, one of the important issues which should be taken into consideration is the use of drugs that may induce DILI in these patients (especially when study protocols are scheduled). Finally, increased surveillance with careful monitoring of serum hepatic enzymes is imperative, especially in hospitalized patients or those with liver comorbidities.

\section{References}

1. Zhu N, Zhang D, Wang W, et al; China Novel Coronavirus Investigating and Research Team. A novel coronavirus from patients with pneumonia in China, 2019. N Engl J Med 2020;382:727-733.

2. European Centre for Disease Prevention and Control. COVID-19 situation update worldwide, as of 1 June 2020. Available from: https://www.ecdc.europa.eu/en/geographical-distribution-2019ncov-cases [Accessed 2 June 2020].

3. Chen N, Zhou M, Dong X, et al. Epidemiological and clinical characteristics of 99 cases of 2019 novel coronavirus pneumonia in Wuhan, China: a descriptive study. Lancet 2020;395:507-513.

4. Wu Z, McGoogan JM. Characteristics of and important lessons from the coronavirus disease 2019 (COVID-19) outbreak in China: summary of a report of 72314 cases from the Chinese Center for disease control and prevention. JAMA 2020 Feb 24 [Epub ahead of print]. doi: 10.1001/jama.2020.2648

5. Xu L, Liu J, Lu M, Yang D, Zheng X. Liver injury during highly pathogenic human coronavirus infections. Liver Int 2020;40:998-1004.

6. Xie H, Zhao J, Lian N, Lin S, Xie Q, Zhuo H. Clinical characteristics of non-ICU hospitalized patients with coronavirus disease 2019 and liver injury: A retrospective study. Liver Int 2020;40:1321-1326.

7. Drosten C, Günther S, Preiser W, et al. Identification of a novel coronavirus in patients with severe acute respiratory syndrome. $N$ Engl J Med 2003;348:1967-1976.

8. Wang $\mathrm{D}, \mathrm{Hu} \mathrm{B}, \mathrm{Hu} \mathrm{C}$, et al. Clinical characteristics of 138 hospitalized patients with 2019 novel coronavirus-infected pneumonia in Wuhan, China. JAMA 2020;323:1061-1069.

9. Chau TN, Lee KC, Yao H, et al. SARS-associated viral hepatitis caused by a novel coronavirus: report of three cases. Hepatology 2004;39:302-310.

10. Li J, Fan JG. Characteristics and mechanism of liver injury in 2019 coronavirus disease. J Clin Transl Hepatol 2020;8:13-17.

11. Chai X, Hu L, Zhang Y, et al. Specific ACE2 expression in cholangiocytes may cause liver damage after 2019-nCoV infection. bioRxiv 2020. doi: 10.1101/2020.02.03.931766

12. Sun J, Aghemo A, Forner A, Valenti L. COVID-19 and liver disease. 
Liver Int 2020;40:1278-1281.

13. Huang Y, Yang R, Xu Y, Gong P. Clinical characteristics of 36 non-survivors with COVID-19 in Wuhan, China. medRxiv 2020.02.27.20029009; doi: https://doi.org/10.1101/2020.02.27.200 29009.

14. Xu XW, Wu XX, Jiang XG, et al. Clinical findings in a group of patients infected with the 2019 novel coronavirus (SARSCov-2) outside of Wuhan, China: retrospective case series. BMJ 2020;368:m606.

15. Shi H, Han X, Jiang N, et al. Radiological findings from 81 patients with COVID-19 pneumonia in Wuhan, China: a descriptive study. Lancet Infect Dis 2020;20:425-434.

16. Guan WJ, Ni ZY, Hu Y, et al; China Medical Treatment Expert Group for Covid-19. Clinical characteristics of coronavirus disease 2019 in China. N Engl J Med 2020;382:1708-1720.

17. Wu J, Liu J, Zhao X, et al. Clinical characteristics of imported cases of COVID-19 in Jiangsu province: a multicenter descriptive study. Clin Infect Dis 2020 Feb 29 [Epub ahead of print]. doi: 10.1093/cid/ ciaa199

18. Huang C, Wang Y, Li X, et al. Clinical features of patients infected with 2019 novel coronavirus in Wuhan, China. Lancet 2020;395:497-506.

19. Cai Q, Huang D, Ou P, et al. COVID-19 in a designated infectious diseases hospital outside Hubei Province, China. Allergy 2020 Apr 2 [Epub ahead of print]. doi: 10.1101/2020.02.17.20024018

20. Cao W, Shi L, Chen L, Xu X, Wu Z. Clinical features and laboratory inspection of novel coronavirus pneumonia (COVID-19) in Xiangyang, Hubei. medRxiv 2020. doi: 10.1101/2020.02.23.20026963

21. Yang X, Yu Y, Xu J, et al. Clinical course and outcomes of critically ill patients with SARS-CoV-2 pneumonia in Wuhan, China: a single-centered, retrospective, observational study. Lancet Respir Med 2020;8:475-481.

22. Mo P, Xing Y, Xiao Y, et al. Clinical characteristics of refractory COVID-19 pneumonia in Wuhan, China. Clin Infect Dis 2020 Mar
16 [Epub ahead of print]. doi: 10.1093/cid/ciaa270

23. Zhou F, Yu T, Du R, et al. Clinical course and risk factors for mortality of adult inpatients with COVID-19 in Wuhan, China: a retrospective cohort study. Lancet 2020;395:1054-1062.

24. Goyal P, Choi JJ, Pinheiro LC, et al. Clinical characteristics of COVID-19 in New York City. N Engl J Med 2020 Apr 17 [Epub ahead of print]. doi: 10.1056/NEJMc2010419

25. Luo S, Zhang X, Xu H. Don't overlook digestive symptoms in patients with 2019 novel coronavirus disease (COVID-19). Clin Gastroenterol Hepatol 2020;18:1636-1637.

26. Fan Z, Chen L, Li J, et al. Clinical features of COVID-19-related liver functional abnormality. Clin Gastroenterol Hepatol 2020;18:1561-1566.

27. Pan L, Mu M, Yang P, et al. Clinical characteristics of COVID-19 patients with digestive symptoms in Hubei, China: a descriptive, cross-sectional, multicenter study. Am J Gastroenterol 2020; 115:766-773.

28. Jin X, Lian JS, Hu JH, et al. Epidemiological, clinical and virological characteristics of 74 cases of coronavirus-infected disease 2019 (COVID-19) with gastrointestinal symptoms. Gut 2020;69:10021009.

29. Cai Q, Huang D, Yu H, et al. COVID-19: Abnormal liver function tests. J Hepatol 2020 Apr 13 [Epub ahead of print]. doi: 10.1016/j. jhep.2020.04.006.

30. Qi X, Liu C, Jiang Z, et al. Multicenter analysis of clinical characteristics and outcome of COVID-19 patients with liver injury. J Hepatol 2020 Apr 17 [Epub ahead of print]. doi: 10.1016/j. jhep.2020.04.010.

31. Zhang B, Zhou X, Qiu Y, et al. Clinical characteristics of 82 death cases with COVID-19. medRxiv 2020; in press. https://doi. org/10.1101/2020.02.26.20028191

32. Bangash MN, Patel J, Parekh D. COVID-19 and the liver: little cause for concern. Lancet Gastroenterol Hepatol 2020;5:529-530.

33. Boettler T, Newsome PN, Mondelli MU, et al. Care of patients with liver disease during the COVID-19 pandemic: EASL-ESCMID position paper. JHEP Rep 2020;2:100113. 